\title{
Carta aos editores
}

\section{The value of publishing negative results from a randomized controlled trial: the rosenheck's study}

\section{Mr Editor,}

In the last few years, attention has been givendrawn tofor the problem ofwith publications bias: itit is well established that papers with negative results (when the null hypothesis is not refused) are less likely to be published in scientific journals than those with results favoring a given intervention. ${ }^{1}$

The paper published in November 23rd, 2003 in JAMA by Rosenheck et al. ${ }^{2}$ reports negative results (no differences) in randomized clinical outcomes when comparing for the comparison of olanzapine and haloperidol in combination with benzotropine tofor treating schizophrenia. These findings, however, do not agreefit with the main results of a Cochrane Systematic Review, which currently included 20 randomized controlled trials (RCTs). In this Review, olanzapine has advantages whenas compared to First Generation Antipsychotics in terms of clinical improvement in negative symptoms. ${ }^{3}$

In Rosenheck's trial, offrom a total of 4386 subjects were screened, andand 2141 were eligible for inclusion, and only 309 were randomized. This restrictive inclusion enrollment process limits the generalizability of the study's findings and resulted in a sample of chronic patients with longer duration of diseases, aged 45 years in average (in olanzapine trials, the mean age of patients is around 35 years). In a more chronic population with schizophrenia it is expected that smaller differences between two treatments are to can be found. ${ }^{4}$ Therefore, lack of statistical power could be another explanation for their negative results.

However, the critical point in this paper is a missing and simple principle: just because of chanceit is expected that some trials will find no significant differences in one or more outcome measures only by chance. According to the Central Limit Theorem, ${ }^{5}$ it is expected that $5 \%$ of the total set of studies will find extreme results (more than two standard deviations from the mean), or $3216 \%$ will stand beyond about one standard deviation from the actual mean.

It is crucial that high impact journals like JAMA publish trials with negative results - readers can have then a real sense about how different samples of patients (in RCTs) can produce different results. If a pharmaceutical company sponsors the trial, this is even crucial. General rules of medical statisticals, such as estimations of samples, heterogeneity of populations, and the selection process, must always be considered. For the best care of individual patients, when assessing scientific information, negative results should be more than welcome by both publishers and readers, but theirits conclusions need to be considered in a more comprehensivewide view, in the context of other similar studies.

Conflito de interesses: Maurício Silva de Lima é Gerente Médico de Neurociências do Laboratório Eli Lilly do Brasil.

Maurício Silva de Lima Federal University of Pelotas and Catolic University of Pelotas, Brazil; Institute of Psychiatry, London, United Kingdon; and Eli Lilly Brasil Bernardo Garcia de Oliveira Soares Cochrane Center of Brasil and Federal University of São Paulo, Brazil

\section{References}

1. Sutton AJ, Duval SJ, Tweedie RL, Abrams KR, Jones DR. Empirical assess ment of effect of publication bias on meta-analyses. British Medical Journal 2000;320(7249):1574-7.

2. Rosenheck R, Perlick D, Bingham S, Liu-Mares W, Collins J, Warren S, et al. Effectiveness and cost of Olanzapine and Haloperidol in the treatment of schizophrenia. A randomized controlled trial. JAMA 2003;290:2693-702. 3. Breier A, Schreiber JL, Dyer J, Pickar D. National Institute of Mental Health longitudinal study of chronic schizophrenia. Prognosis and predic tors of outcome. Arch Gen Psychiatry 1991;48(3):239-46.

4. Kirkwood B. Confidence interval for a mean. In: Kirkwood B. Essential of Medical Statistics. London: Blackwell Scientific Publications; 1988.

\section{Methodological considerations on the com- parison of first and second generation antipsychotics}

Mr. editor,

Drs. Silva de Lima and Garcia de Oliveira Soares suggest that, in view of the large number of studies of second generation antipsychotics (SGAs) that have been conducted over the years, our finding of limited benefit for olanzapine as compared to haloperidol $m$ ay be attributable to chance. This assertion rests on the assumption that all the studies of SGAs, including ours, used the same methodology. We believe that it is such methodological differences that explain the differences in results. The most revealing outcome difference between our study and the International Collaborative Trial (ICT), ${ }^{1}$ the major study of olanzapine, is that while adherence to olanzapine was the same in both studies adherence to haloperidol was far superior in ours, almost certainly because we used prophylactic anticholinergics with haloperidol while the ICT use anticholinergics on an "as-needed" basis, for only $50 \%$ of patients.

While this could clearly explain the lack of differences in Parkinsonian side effects, could it also explain the difference in 
symptoms? First it should be pointed out that the ICT found no significant difference between olanzapine and haloperidol on positive symptoms, but only on negative symptoms and depression. ${ }^{1}$ Furthermore, as we pointed in our paper these symptom findings may have been an artifact of two serious methodological flaws that appear in the ICT and many studies of SGAs: 1) failure to continue to collect outcome data until the end of the trial on all subjects including those who changed medication, combined with 2) use of last observation carried forward (LOCF) analysis.

In addition, there is an extensive literature showing that one extrapramidal symptom, akinesia, can be indistinguishable from negative symptoms of schizophrenia and depression. ${ }^{2}$ and this EPS side effect could explain the differences between our study and the ICT. In a recent meta-analysis, Leuct found SGAs to have lower relapse rates than first generation antipsychotics (FGAs). ${ }^{3}$ However, $91 \%$ of the studies in this meta-analysis used haloperidol as the comparator and only $20 \%$ of these prescribed prophylactic anticholinergics. Reanalysis of these data shows that only when haloperidol was used without prophylactic anticholinergics there was risk of relapse, all cause failure and early termination less with SGAs than with haloperidol.

But could this meta-analysis of only 10 studies be generalizable to the many other studies of SGAs? A much larger meta-analysis involving 124 studies by Davis et al. ${ }^{4}$ showed that two-thirds of all controlled trials of SGAs used haloperidol without prophylactic anticholinergics as the comparator, and thus were likely to have been seriously biased as noted above.

Taking all of the more than 130 studies reviewed in three large meta-analyses together, ${ }^{3-5}$ about two-thirds of the studies gave an unfair advantage to SGAs by comparing them with haloperidol without prophylactic anticholinergics, while the remainder, using Iow potency FGAs, did not find a robustly significant advantage for SGAs.

Drs. Silva de Lima and Garcia de Oliveira Soares also suggests that our study was under-powered and unrepresentative. We presented a power analysis in the methods section showing the first claim to be unlikely. We have also reanalyzed the data using only younger subjects and found the same results.

Robert Rosenheck New Haven, CT

\section{References}

1. Tollefson GD, Beasley Jr CM, Tran PV, et al. Olanzapine versus haloperi $\mathrm{dol}$ in the treatment of schizophrenia and schizoaffective and schizo phreniform disorders: results of an international collaborative trial. Am J Psychiatry 1997; 154:457-65.

2. Van Putten T, Marder SR. Behavioral toxicity of antipsychotic drugs. J Clin Psychiatry 1987;48 Suppl:13-9.

3. Leucht S, Barnes TR, Kissling W, Engel RR, Correll C, Kane JM. Relapse prevention in schizophrenia with new-generation antipsychotics: a sys tematic review and exploratory meta-analysis of randomized, controlled trials. Am J Psychiatry 2003;160:1209-22.

4. Davis JM, Chen N, Glick ID. A meta-analysis of the efficacy of second-gen eration antipsychotics. Arch Gen Psychiatry 2003 60:553-64.

5. Leucht S, Wahlbeck K, Hamann J, Kissling W. New generation antipsy chotics versus low-potency conventional antipsychotics: a systematic review and meta-analysis. Lancet 2003;361:1581-9.

\section{Use of gabapentin in group B - DSM-IV per- sonality disorders}

Mr. editor,

Many professionals are skeptical regarding the treatment of personality disorders (PD) for considering it protracted and unsatisfactory. ${ }^{1}$

The therapeutical refractoriness of PD cannot be deduced from the diagnostic label in itself, but from the assessment of all the factors related to the subject's personality and global functioning. The identification of psychopathological aspects related to excitability, mood pattern, emotional lability and tolerance to frustrations, are important in the treatment, and may be accessible to pharmacological and psychotherapeutic approaches and to the psychosocial rehabilitation. The adequate development of social feelings, as a capability of considering the other, and the ethical awareness are decisive factors for that.

The PD outpatient clinic at the Psychiatric Institute of HC-FMUSP (IPq) has started its activities at the beginning of 1999 aiming the early intervention on those patients, in order to prevent the delinquent behavior, very common in the life history of those subjects. From June 2002 up to June 2003, 137 PD patients were seen at IPq's outpatient clinics. Of these, 40 [29.19\%] were seen at the PD outpatient clinic.

It was observed that many of them had a long history of psychiatric consultations and hospitalizations, without improvement, besides representing a burden to their families and to society.

The main complaints were related to aggressiveness, hostility, impulsiveness, immediatism, irresponsibility, suggestibility, lack of introspection, affective and working instability, trend to lie frequently, drug use (without dependence), obstinate behavior and insensitivity regarding the others. Some of them had already committed some crimes against people, such as murder attempt, robbery, rape and corporal lesion, rarely with legal consequences due to lack of denunciation.

Several neuropsychopharmacological studies suggest a biological substrate for PD, what could be reduced by psychopharmacoIogical intervention. ${ }^{2}$ Gabapentin was chosen due to its probable inhibitory effect in the brain neurotransmission, ${ }^{3}$ reducing the psychic hyper-excitability, different from that seen in mood disorders.

The aim of this observational study was to assess the behavioral improvement in group B PD (DSM IV) patients with the use of gabapentin.

The diagnosis was established using international criteria (ICD10;DSM-IV), and, in some cases, using personality assessment instruments (Rorschach Proof and PCL-R). 4,5 The intervention was psychotherapeutical and pharmacological.

Twenty-nine patients have been treated (8 with antisocial PD; 13 impulsive type; 7 histrionic type and 1 narcissistic), with the maximal dose of gabapentin $1200 \mathrm{mg} /$ day, alone or concomitantly with other drugs (neuroleptics, mood stabilizers and benzodiazepines). In 23 of them (79.9\%) it was verified, through the reports of patients and their people in charge, an improvement in the initial picture after 6 weeks of treatment, with decrease of aggressiveness, impulsivity, antisocial behavior and drug abuse. There was also an improvement in the concentration and introspection capabilities and higher interest in productive activi- 